\title{
México 19S: ¿Punto de quiebre o de partida?
}

"Un fuerte terremoto destruye de inmediato las asociaciones más viejas: el mundo,
el símbolo mismo de todo lo que es sólido, se ha movido bajo nuestros pies como
una capa sobre un líquido; un segundo de tiempo le ha transmitido a la mente una
extraña idea de inseguridad, que nunca habría surgido con horas de reflexión".

Charles Darwin

"Un huracán de pensamientos se libera después de cada nuevo entendimiento. Éste a su vez resulta en un terremoto de premisas.

Estos son los desastres naturales que remodelan el espíritu".

Vera Nazarian

\section{$19 S$}

Amables lectores, debí haber escrito esta Editorial a principios de septiembre, pero la procrastinación que frecuentemente nos afecta me llevó a diferir el sentarme ante la página en blanco durante algunas semanas, hasta que el 195 nos impactó en la Ciudad de México. Ahora que escribo estas líneas es imposible no tocar el tema; si así lo hiciera, estaría faltando al respeto a los millones de personas afectadas por el terremoto, cuyos efectos continuamos procesando. En estos días he leído docenas de editoriales en los medios, que abordan el tema desde muchas perspectivas. Intentaré hacerlo con sensibilidad, sin restar énfasis en las lecciones a aprender, y situando algunos de los manuscritos que aparecen en este número de la Revista Digital Universitaria $(R D U)$ en un panorama de utilidad y optimismo. Es fascinante cómo Charles Darwin capturó en unas líneas el brutal efecto de unos cuantos segundos en la vida de las personas, y cómo emerge esta profunda sensación de inseguridad que, como dice la cita: "nunca habría surgido con horas de reflexión". En un breve intervalo de tiempo el contexto de muchísimas personas cambió radicalmente, y día a día nos preguntamos cómo podemos contribuir con nuestro grano de arena en el amplio horizonte de acciones posibles. Creo firmemente que las instituciones educativas y académicas, como la nuestra, están en una situación idónea para aportar ideas y acciones que promuevan el regreso a esa fluida y efímera situación que, a falta de otro descriptor, llamamos "normalidad".

El 19 de septiembre por la mañana, tuvimos una reunión de trabajo con nuestro Rector, el Dr. Enrique Graue Wiechers, otros rectores de universidades públicas y privadas de varios estados de la República, y con académicos y representantes de dichas entidades, para dar continuidad a una iniciativa de innovación educativa. Los detalles de la constitución de este grupo interuniversitario los comentaremos en el siguiente número de la RDU. Baste decir que ese día, en el agradable escenario de la Unidad de Seminarios "Ignacio Chávez" de la UNAM, se solidificó la gestación de una iniciativa que representa un poderoso ejemplo de trabajo en 
Revista Digital Universitaria

Vol. 18, Núm. 7, septiembre-octubre 2017

equipo, con creatividad y rigor académico, para desarrollar intervenciones en beneficio de los estudiantes de educación superior. Al final de esa productiva sesión, todos los presentes nos dirigimos a nuestras instituciones para participar en el macrosimulacro programado a las 11 de la mañana, que transcurrió "sin novedad". Las personas que no habían nacido en 1985 y aquellos que ya teníamos consciencia en esa fecha, colaboramos de manera rutinaria en el proceso de este ejercicio, algunos sin tener muy claro de qué se trata este "ritual", al que nos hemos acostumbrado en los últimos 32 años. Después de caminar ordenadamente a nuestros puntos de reunión y de platicar trivialidades, regresamos a nuestras áreas de trabajo muy quitados de la pena.

Sobra decir que todos los que vivimos en la sufrida Ciudad de México, al sentir el movimiento telúrico a las 13:14:40 horas del 19S, vivimos unos instantes de angustia y miedo, por el riesgo de morir, propio y de nuestros seres queridos, o de perder aquellos bienes materiales que, si bien son secundarios, tanto trabajo nos ha costado adquirir. Cada uno de nosotros tiene multitud de historias que contar sobre lo que experimentó en esos aciagos momentos, que nos hicieron -y nos siguen haciendo - repensar el total de nuestras prioridades vitales y personales. Con el paso de los días, la cifra creciente de personas fallecidas y heridas, de casas y edificios dañados, el retorno a la rutinaria normalidad se ve cada vez más lejano.

\section{¿Qué aporta este número de la RDU?}

Los manuscritos que integran este número de la revista ofrecen un caleidoscopio de áreas del conocimiento con intensa vigencia, originándose en diversas perspectivas del quehacer universitario y ofreciendo potenciales soluciones a problemas que aquejan a la sociedad moderna. A continuación, comentaré brevemente algunos de ellos, con la perspectiva particular a la que nos obliga la situación actual del país:

- "¿Qué tan derecho es el derecho a la educación en México?" ¡Qué tema tan ad hoc para empezar! Además de ser uno de los tópicos más apasionantes e importantes del discurso y acción social actuales, lo que nos llevó a agregar apartados educativos en la RDU para explorar estos temas desde diversas ópticas, es una de las esferas más directamente afectadas por el 19S. El análisis de este derecho, a la luz de los cuatro principios de los derechos humanos descritos por la autora: universalidad, indivisibilidad, interdependencia y progresividad, en el contexto de los desastres naturales, nos debe llevar a reflexionar profundamente sobre el tema y a hacer lo posible para que estos principios no sean sólo palabras vacías en nuestra Carta Magna. La pérdida de vidas en centros educativos, de estudiantes (niños, jóvenes y adultos), docentes, administrativos y trabajadores, así como los esfuerzos colectivos de la sociedad y las dependencias oficiales para rescatar con vida a los sobrevivientes, ha sido uno de los puntos más poderosos emocionalmente en este catastrófico evento. ¿Cómo borrar de nuestras mentes las imágenes y videos de las instalaciones educativas destruidas y gravemente dañadas?, ¿la expresión de angustia, miedo y dolor de los testigos de estos hechos y de los familiares de los afectados?, dicen que el tiempo sana todas las heridas, pero éstas son demasiado profundas como para no dejar cicatrices permanentes en nuestra psique individual y colectiva.

Universidad Nacional Autónoma de México, Coordinación de Desarrollo Educativo e Innovación Curricular (CODEIC) Este es un artículo de acceso abierto bajo la licencia de Creative Commons 4.0 
Revista Digital Universitaria

Vol. 18, Núm. 7, septiembre-octubre 2017

Uno de los efectos inmediatos del desastre fue la cancelación de clases en todos los niveles educativos, que a 10 días del evento distan mucho de regresar a la normalidad, sobre todo en la educación básica. Es entendible la preocupación de autoridades, padres de familia y de los propios estudiantes por la seguridad; pero es menester reflexionar sobre los múltiples efectos que esto conlleva: al no asistir los niños a la escuela, las madres y padres que trabajan se ven en la encrucijada afectiva, logística y financiera de qué hacer con ellos mientras tanto, ¿quién los cuidará?, ¿quién les dará el apoyo psicológico necesario?, ¿quién modulará las noticias del terremoto y sus consecuencias para que las entiendan en su justa dimensión?, ¿cómo cubrir el gasto extra en caso de que haya que pagar por su cuidado?, ¿cómo compensar la pérdida de muchos días de educación formal?, ¿quién les va a dar la "escuela en casa"? Todas estas preguntas y otras requieren respuestas inmediatas, y no siempre se obtiene el apoyo necesario, efectivo y expedito de las instituciones escolares, docentes, o se carece de un andamiaje familiar y de amistades adecuado. Todas las mamás que conozco están angustiadas en el trabajo, pensando cómo resolver estos retos y actuar en consecuencia, con los potenciales efectos en su desempeño laboral. Es momento de exhibir sensibilidad y compañerismo con nuestras colegas que se encuentran en esta situación, en el espacio de trabajo y en el entorno familiar, y que hagamos un esfuerzo porque esta actitud de soporte organizacional a las personas que tienen hijos se convierta en algo permanente.

En una exhibición de resiliencia impresionante, ciudadanos de todas las edades y todas las condiciones sociales, no sólo están saliendo adelante, sino que se están creciendo al reto. Los estudiantes adolescentes y adultos que se encuentran en los niveles de educación media y superior, nos han dado una gran lección con su actitud y sus acciones, ayudando con todas sus fuerzas, sin mirar a quién y sin esperar recompensa alguna sino la satisfacción de ayudar al prójimo. iQué esta conducta permanezca y contagie a todos los estratos etarios!

Otro aspecto educativo a considerar, es ser testigos de cómo las vivencias intensas de este tipo generan un aprendizaje profundo y extremadamente significativo. A los que nos dedicamos a la docencia, lo que hemos atestiguado durante y después del temblor debe ponernos a pensar sobre la importancia relativa de la educación formal en el interior de las paredes institucionales. ¿Cuándo se aprende más, cuando se lee algo, se realiza un ejercicio de simulación artificial, o cuando se enfrenta un problema real que requiere soluciones inmediatas de consecuencias imprevistas? Estoy seguro que los médicos jóvenes, los estudiantes de pre y posgrado de ingeniería y arquitectura, entre muchos otros, aprendieron (y se transformaron) mucho más profundamente con esta experiencia que con cualquier ejercicio, tarea o solución de problemas en papel o en computadora. Por supuesto, no quiero decir que es mejor que haya desastres para que ocurra el aprendizaje, sino que este tipo de vivencias debe obligarnos a los docentes a poner los pies en la tierra y a no sobreestimar el efecto educativo de las experiencias curriculares excesivamente planeadas y microcontroladas, como nuestras conferencias tradicionales o los programas académicos de las asignaturas que en el papel se ven muy bien. Reflexionen aquellos que tengan responsabilidades docentes, ¿qué haremos con la disminución de días de clases en nuestro calendario académico, que se ha visto irremediablemente afectado por la catástrofe? La solución simplista de "reponer horas de clase" para "cubrir todo el programa", sin 
Revista Digital Universitaria

Vol. 18, Núm. 7, septiembre-octubre 2017

tener en cuenta el contexto profundamente transformado de nuestra sociedad, no debe ser la respuesta predominante. Se requiere creatividad, innovación, trabajo en equipo y compromiso, tanto de docentes como de estudiantes, así como de las autoridades responsables de los centros educativos.

Una de las lecciones más importantes que nos deja el terremoto, es la importancia de la educación en la prevención, actuación debida durante y después de desastres mayores, para disminuir en lo posible los daños y las pérdidas humanas. Si uno de los factores que inciden en el aprendizaje es la repetición, la madre naturaleza se ha encargado de recordarnos, una y otra vez, lo importante que es poseer las habilidades y competencias para tener un desempeño adecuado al enfrentar desastres como un huracán o un terremoto, más allá del conocimiento teórico. Es curiosa la ausencia en el currículo formal de muchas de nuestras instituciones educativas, de la enseñanza y evaluación de las habilidades mínimas para confrontar este tipo de desastres naturales. Aunque en Internet y en la literatura científica existe una gran cantidad de recursos para aprender los conceptos básicos y avanzados sobre terremotos y otros desastres, frecuentemente no los revisamos hasta después del evento. Salimos debiéndole, en los hechos, a la cultura de prevención de la que tanto hablamos. En las pláticas de café en estos días (en las que es inevitable hablar del tema), todos nos hemos convertido en "expertos" en la escala de Richter, epicentros, diferencias entre oscilatorio y trepidatorio, Gales, la placa de Cocos, etcétera, ojalá este caudal de conocimientos permanezca y nos motive a profundizar en lo que realmente podemos hacer para tener respuestas más efectivas ante las catástrofes.

El cruel recordatorio de la naturaleza, que coincidió de manera inexplicable con el día 19 de septiembre, nos hizo rememorar vívidamente la experiencia que tuvimos en nuestra ciudad con el terremoto de 1985. Recordamos que también en esa época nos convertimos en "terremotólogos" de la noche a la mañana. A pesar de que muchas cosas se han hecho mejor en los últimos 32 años, aún nos falta muchísimo por realizar en el terreno educativo, creo que debemos "hacer de la necesidad virtud". Incorporemos en nuestros currículos formales, vividos y ocultos los conceptos más relevantes para sobrevivir a los desastres y desaparezcámoslos del currículo nulo (concepto que se refiere a temas de estudio no enseñados, o que siendo parte del currículo no tienen aplicabilidad ni utilidad aparente, considerándose como contenido superfluo). Invito a los lectores a que tengamos en nuestras listas de "favoritos" recursos educativos confiables sobre estos temas, de los que existen muchos en la red (OMS, OPS, National Geographic, NEHRP, UNESCO) (1-5). Existen también aplicaciones para dispositivos móviles que, utilizando videojuegos, pueden enseñar a tener conciencia sobre la importancia de estar preparado para situaciones de emergencia, un ejemplo es Tanah: contra los terremotos y tsunamis desarrollado por la UNESCO (ㅁ). Una de las fuentes de recursos más interesante sobre el tema es el International Network for Education in Emergencies (INEE), una red internacional de más de 13000 miembros individuales y 130 organizaciones en 190 países (ㄱ). Los miembros de esta organización son personas interesadas en el tema (docentes, estudiantes, investigadores), organizaciones no gubernamentales, ministerios de educación y agencias de las Naciones Unidas que, de manera voluntaria, han unido esfuerzos para trabajar apoyando la educación durante emergencias (Shroder) (ㄱ). Creo que vale la pena integrarse a este tipo de iniciativas, para que se sigan desarrollando e incrementen su capacidad de acción. 
Revista Digital Universitaria

Vol. 18, Núm. 7, septiembre-octubre 2017

En conclusión, como han afirmado los expertos en el tema: "la educación ha sido clave para disminuir la mortalidad en los terremotos" (nopoff) (ㅇ).

- "Científico de datos: codificando el valor oculto e intangible de los datos". Uno de los cambios paradigmáticos más importantes del siglo XXI ha sido el advenimiento del concepto de Big data (volúmenes masivos de datos), y los sofisticados métodos analíticos utilizados para entender con precisión complejos fenómenos biológicos, físicos y sociales que antes eran imposibles de explorar. El artículo describe este fascinante campo y la nueva casta de científicos pionera en el área, los data scientists (científicos de datos), una combinación fascinante de pericia en diferentes disciplinas que rápidamente se está convirtiendo en una de las profesiones más solicitadas.

Sobre el tema que nos ocupa, el uso de Big data podría convertirse en uno de los "cambiadores de juego" en el escenario de predicción de los desastres. Estos días hemos oído en todos los noticieros y entrevistas a expertos, que es imposible predecir los terremotos y, en consecuencia, que tenemos que aprender a vivir en la incertidumbre (sabiendo que la Ciudad de México se encuentra en un área sísmica, y que tarde o temprano volveremos a repetir la experiencia vivida recientemente). Estas afirmaciones generalmente no tranquilizan mucho a la sociedad, aunque desde el punto de vista técnico parece ser que este tipo de fenómenos son inherentemente impredecibles (Marr, 2015) (9). El uso de "terremoto" y "predicción" en la misma oración inmediatamente genera escepticismo, principalmente en la comunidad científica. El pronóstico de los terremotos es el "Santo Grial" de la sismología, y por muchas décadas ha sido buscado por los investigadores del área, sin éxito contundente (Marr, 2015 y 2016) (-10). Sin embargo, con el uso de la "magia" de la analítica de Big data, algunas organizaciones (http://www.terraseismic.com y otras) están utilizando los volúmenes masivos de datos generados de diversas fuentes (como los producidos por la red de satélites que circunda el planeta) para intentar predecir, con cierto grado de precisión, terremotos importantes que tengan potencial de causar daño (Singh y Haraksingh, 2016; Kannan, 2014; Sneed, 2017) (10-13). Es importante señalar que aún no se ha resuelto el problema, que los académicos expertos en este tema recomiendan ser muy prudentes en la interpretación de la literatura (científica y de divulgación) que reporta estos interesantes hallazgos, y que no echemos las campanas al vuelo por artículos aislados o trabajos de empresas que tienen un conflicto de interés intrínseco al publicitar la efectividad de sus métodos e instrumentos. Sin embargo, el uso de métodos cada vez más sofisticados de inteligencia artificial y machine learning (aprendizaje de máquinas), podría ofrecer nuevos caminos para llegar a resolver este "acertijo imposible" (14).

Independientemente de lo lejos que estemos de tener un sistema de predicción de terremotos confiable, rápido y disponible para todos los países, creo que estos últimos eventos catastróficos deben ser una ruidosa alarma, un semáforo rojo, una llamada de atención impostergable a nuestros gobernantes, para que dediquen los recursos humanos y financieros que sean necesarios al desarrollo de la investigación científica. Ésta será la mejor manera de seguir avanzando en la prevención y detección temprana de los desastres naturales, en lugar del actual sentimiento de inevitabilidad y vulnerabilidad que prevalece. Por lo pronto, utilicemos los recursos globales que se han desarrollado, como los mapas de áreas afectadas por el terremoto proporcionadas por la NASA a las autoridades nacionales y a la comunidad en general, para ayudar a identificar las zonas dañadas en la Ciudad

Universidad Nacional Autónoma de México, Coordinación de Desarrollo Educativo e Innovación Curricular (CODEIC) Este es un artículo de acceso abierto bajo la licencia de Creative Commons 4.0 
Revista Digital Universitaria

Vol. 18, Núm. 7, septiembre-octubre 2017

de México (disponibles en: https://www.jpl.nasa.gov/spaceimages/details.php?id=pia21963). El vaso de la tecnología no está totalmente lleno, pero tenemos la obligación moral de tener consciencia de estos recursos, ya que no utilizarlos se convierte en un desastre en sí mismo.

- "Programa Adopte un Talento: un vínculo entre la comunidad científica y los niños". Este artículo es extraordinariamente inspirador. No hay tarea más importante en la especie humana que la educación, ya que de ella se desprende todo lo positivo que podemos realizar en nuestro transitorio paso por el planeta. El programa PAUTA es una iniciativa innovadora, que pretende promover la inquietud científica en nuestro más preciado tesoro, la niñez mexicana. La metodología utilizada en el programa ha tenido resultados fascinantes, por lo que esperamos que en un futuro cercano haya iniciativas similares en otras instituciones. Debemos crear ese catalizador tan necesario en nuestro país: una comunidad científica competente, creativa, crítica, proactiva y propositiva, que contribuya a resolver los problemas ingentes de nuestra sociedad. Necesitamos más geólogos, sismólogos, paleosismólogos, divulgadores de la ciencia que expliquen con claridad estos fenómenos, y una sociedad dotada con las habilidades de pensamiento crítico suficientes para interpretar, cuestionar y aplicar los conocimientos generados por el gremio científico. La Universidad Nacional Autónoma de México, a través del Servicio Sismológico Nacional del Instituto de Geofísica, es el referente nacional en los temas de sismos, temblores y terremotos, es un hecho que debemos continuar impulsando su desarrollo e incrementando la red de instituciones nacionales e internacionales que son capaces de analizar y explicar estos fenómenos.

\section{¿Procrastinar?, ¡no!}

En este número de la revista aparecen también manuscritos de instituciones educativas, estudiantes y docentes, sobre los temas de la aplicación de las ciencias "-ómicas", las partículas subatómicas y las supercomputadoras, la experiencia digital de un educando y su impacto en la necesidad formativa, y la importancia de la formación tecnológica en la cultura científica. Invitamos a los lectores asiduos (iy a los nuevos!) de nuestra publicación a explorar estos interesantes temas, no solo para incrementar su acervo cultural, sino para poner manos a la obra en la dura tarea de la recuperación de nuestro país. Con dolorosa frecuencia escuchamos que los mexicanos tenemos nuestra propia versión de "no dejes para mañana lo que puedas hacer hoy", transformándolo en: "no hagas hoy lo que puedas hacer mañana". Procrastinar es uno de los verbos que debemos intentar erradicar de nuestro léxico cotidiano nacional, para enfrentar con eficacia nuestras duras realidades.

Editor en Jefe Melchor Sánchez Mendiola Facultad de Medicina, UNAM 
Revista Digital Universitaria

Vol. 18, Núm. 7, septiembre-octubre 2017

\section{Referencias}

1. Organización Mundial de la Salud. Acción Sanitaria en las Crisis Humanitarias. Terremotos. http://www.who.int/hac/techguidance/ems/earthquakes/es/ (Accesado el 29 de septiembre de 2017).

2. Organización Panamericana de la Salud. Departamento de Emergencias en Salud. http://www.paho.org/disasters/?lang=es (Accesado el 29 de septiembre de 2017).

3. National Geographic. Education. Teaching resources. Eartquakes 101. https://www. nationalgeographic.org/media/earthquakes-101-wbt/ (Accesado el 29 de septiembre de 2017).

4. National Earthquake Hazards Reduction Program. http://www.nehrp.gov/ (Accesado el 29 de septiembre de 2017).

5. UNESCO. Educación en Contextos Emergencia. http://www.unesco.org/new/es/quito/ education/educacion-en-emergencia/guias-para-docentes/ (Accesado el 29 de septiembre de 2017).

6. UNESCO. Tanah. Aplicación contra terremotos y tsunamis. http://www.unesco.org/ new/es/quito/education/educacion-en-emergencia/tanah-contra-los-terremotos-ytsunamis/ (Accesado el 29 de septiembre de 2017).

7. INEE (International Network for Education in Emergencies). http://www.ineesite.org/ en/ (Accesado el 29 de septiembre de 2017).

8. Shroder, J. (2014). In Chile's earthquake, education was key to low mortality. Understanding how to cope with megaquakes is essential for survival. Elsevier Connect. https://www.elsevier.com/connect/in-chiles-earthquake-education-was-key-to-lowmortality (Accesado el 29 de septiembre de 2017).

9. Knopoff, L. (1996). Earthquake prediction: the scientific challenge. Proceedings of the National Academy of Sciences of the United States of America, 93(9), 3719-3720. https:// www.ncbi.n/m.nih.gov/pmc/articles/PMC39427/

10. Marr, B. (2015). Big Data: Saving 13,000 Lives A Year By Predicting Earthquakes? Forbes. https://www.forbes.com/sites/bernardmarr/2015/04/21/big-data-saving13000-lives-a-year-by-predicting-earthquakes/\#2a91cdb5d8da (Accesado el 29 de septiembre de 2017).

11. Marr, B. (ed) (2016) Terra Seismic: Using Big Data to Predict Earthquakes, in Big Data in Practice: How 45 Successful Companies Used Big Data Analytics to Deliver Extraordinary Results, John Wiley \& Sons, Ltd, Chichester, UK. doi:10.1002/9781119278825.ch39 http://onlinelibrary.wiley.com/book/10.1002/9781119278825

12. Singh, D., Haraksingh, I. (2016) Earthquakes can be Predicted. J Geol Geophys, 5:255. doi:10.4172/2381-8719.1000255 https://www.omicsonline.org/peer-reviewed/earthquakes-can-be-predicted-79790.html 
Revista Digital Universitaria

Vol. 18, Núm. 7, septiembre-octubre 2017

13. Kannan, S. (2014) Improving Innovative Mathematical Model for Earthquake Prediction. J Geol Geosci, 3:168. doi:10.4172/2329-6755.1000168 https://www.omicsonline. org/open-access/improving-innovative-mathematical-model-for-earthquake-prediction-2329-6755.1000168.php?aid=28150

14. Sneed, A. (2017). Can Artificial Intelligence Predict Earthquakes? Scientific American https://www.scientificamerican.com/article/can-artificial-intelligence-predict-earthquakes/ (Accesado el 29 de septiembre de 2017).

\section{Cómo citar este artículo}

* Sánchez Mendiola, Melchor (2017). México 19S: ¿Punto de quiebre o de partida?, Revista Digital Universitaria (RDU), vol. 18, núm. 7, septiembre-octubre. Recuperado de http://dx.doi.org/10.22201/ codeic.16076079e.2017.v18n7.a0. 